\title{
Yield Improvement of Air-Fed Ozonizer Using Shorter Pulsed Discharges
}

\author{
Sho Okada, ${ }^{a}$ Takao Namihira, ${ }^{\mathrm{b}}$ \& Douyan Wang ${ }^{\mathrm{b}, *}$ \\ aDepartment of Electrical Materials and Engineering, University of Hyogo, Japan; 'bnstitute of \\ Industrial Nanomaterials, Kumamoto University, Japan \\ *Address all correspondence to: Douyan Wang, Institute of Industrial Nanomaterials, Kumamoto University, Japan; \\ Tel./Fax: +8196342 3945, E-mail: douyan@cs.kumamoto-u.ac.jp
}

\begin{abstract}
Ozone is well-known for its strong oxidation properties, which are used in many industrial applications. Recently, it is also gaining attention in the fields of biology, agriculture, and medicine. Though most commercial ozonizers work via the dielectric barrier discharge method, there are still some issues that need to be improved, including type of seeding gas, byproducts, and ozone yield. In this research, ozone was generated in air using pulsed discharges with different pulse durations $(50,100$, and $200 \mathrm{~ns})$. The results show that shorter pulsed discharges generate ozone at a higher energy efficiency. This is due to the shorter length of the glow discharge phase in the pulse.
\end{abstract}

KEY WORDS: ozone, ozone concentration, ozone yield, streamer discharge, glow discharge, nonthermal plasma, short duration, coaxial electrode geometry

\section{INTRODUCTION}

Ozone has garnered close attention for use in industry because it has stronger oxidation power than chlorine and consists of only oxygen atoms. Recently, there is also interest in its use in the fields of biology, agriculture, and medicine. Medical applications for ozone include disinfecting medical equipment and hospital linen, and treating infected wounds, circulatory disorders, geriatric conditions, macular degeneration, and viral diseases. This is due to its ability to deactivate bacteria and viruses, stimulate oxygen metabolism, activate the immune system, and so on. ${ }^{1}$ Ozone is usually generated in one of three ways: electrochemically, via ultraviolet rays, or via nonthermal plasma produced by electrical discharges. ${ }^{2}$ For practical applications, nonthermal plasma is the most studied method. The abilities of dielectric barrier discharge (DBD), ${ }^{3-6}$ packed-bed discharge, ${ }^{7}$ and DC corona discharge ${ }^{8}$ to generate nonthermal plasmas have been studied for several years. Commercial ozonizers are most commonly manufactured based on the DBD method. However, improvement of ozone yield is still necessary for this technology to be adapted for a wide variety of applications.

Pulsed discharge has been used in various fields, such as laser excitation, exhaust gas treatment, volatile organic compounds (VOCs) treatment, ozone generation, and water treatment. ${ }^{9}$ In ozone generation, pulsed discharge has some advantages: the wide gap of the discharge electrode, no dielectric loss, and high energy efficiency in plasma processing compared with DC and AC power sources. ${ }^{9-13}$ Another important issue for ozone generation is the gas used to generate ozone, either oxygen or air. Commercial ozonizers are mostly oxygen-based DBD ozonizers. In these systems, an air compressor provides 
compressed air to the pressure swing adsorption (PSA) module to generate oxygen as the raw material. The oxygen is excited with discharge pulses to form a molecule containing three oxygen atoms. This process requires a lot of energy to obtain pure oxygen gas, and therefore ozonizers that use air as the source of oxygen are preferred. However, plasma chemical reactions in air produce not only atomic oxygen and ozone, but also byproducts such as nitrogen oxide. This nitrogen oxide reacts with moisture in the seeding gas to create nitric acid. ${ }^{3}$ If nitric acid accumulates in the DBD narrow discharge area, ozone cannot be generated, and the electrodes will corrode, making it necessary to clean the electrodes regularly or replace them frequently. On the other hand, pulse discharge with a high voltage rise rate enables a wide electrode gap, yielding a large discharge volume, which in turn means ozone can be generated regardless of the byproducts. Compared to oxygen-fed ozonizers, there is less research on air-fed ozonizers. ${ }^{14-16}$ This study investigates yield improvement using short duration pulse discharge for ozone generation.

\section{METHODOLOGY}

\section{A. Pulsed Power Generator}

Figure 1 is a schematic diagram of the pulsed power generator used in this study. The generator consists of a Blumlein line generator and a pulse transformer (PT). The winding ratio of the $\mathrm{PT}$ was fixed at 1:3 (one winding to three windings). The Blumlein line generator consists of two coaxial cables (RG-213/U, Mitsubishi Cable Industries, Japan), each with $50 \Omega$ of impedance. The length of the cable defines the pulsed voltage duration. ${ }^{17}$ Three Blumlein line generators were used in this study, with cable lengths of 5,10 , and $20 \mathrm{~m}$, resulting in pulse durations of 50,100, and $200 \mathrm{~ns}$, respectively. The total impedance of the pulsed power generator was calculated as $900 \Omega(50 \Omega \times 2$ cables $\times 3^{2}$ ). In this study, the DC voltage to the Blumlein line generator was fixed at $20 \mathrm{kV}$ for all experiments. A thyratron was used as a closing switch for the Blumlein line generator. It should be noted that a $1 \mathrm{M} \Omega$ resistor was used as a charging resistor to protect the

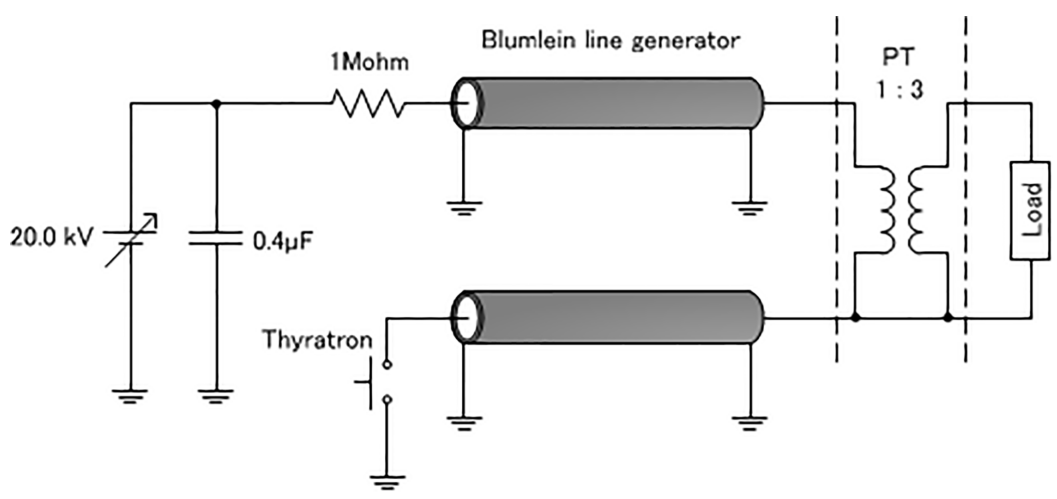

FIG. 1: Schematic diagram of pulsed power generator 
DC power source from reflected energy from the pulse generator. The $0.4 \mu \mathrm{F}$ capacitor acts as a buffer for a stable supply of energy to the Blumlein line generator at a high repetition rate.

\section{B. Ozone Generation}

Figure 2 shows the experimental setup for ozone generation using pulsed discharges. A concentric coaxial cylindrical electrode was used as the discharge reactor. The central wire was made of stainless steel, $0.5 \mathrm{~mm}$ in diameter placed coaxially in a copper cylinder having a $76 \mathrm{~mm}$ internal diameter and a length of $200 \mathrm{~mm}$. It is well known that discharge electrodes have a semipermanent lifetime if there is no arc discharge. It should be mentioned that the electrodes were able to withstand more than 1,000 hours running pulsed discharges. In this study, a positive pulse voltage from the generator was applied to the central wire electrode, and the outer cylinder electrode was grounded.

In ozone generation experiments, dry air consisting of $79 \%$ nitrogen and $21 \%$ oxygen was fed from a gas cylinder into the ozone generation reactor. The gas flow rate was fixed at $1.0 \mathrm{~L} / \mathrm{min}$ at $273 \mathrm{~K}$ and $0.1 \mathrm{MPa}$. The gas treated with repeated discharges was fed to an ozone monitor (V-560, JASCO, Japan) to measure the ozone concentration. The ozone monitor measured the concentration via ultraviolet absorption method at 254 $\mathrm{nm}$, and ozone concentration was calculated using the Lambert-Beer law.

The voltage applied to the electrode from the pulsed power generator was measured using a resistive voltage divider $(1 \Omega: 10 \mathrm{k} \Omega)$ that was connected between the central wire and the ground. The discharge current through the reactor was measured using a current transformer (Pearson current monitor model 2878, Pearson Electronics, USA) that was placed on the return line to ground. The signals from the voltage divider and current transformer were recorded by a digital oscilloscope (Infiniium 54845A, Agilent Technologies, USA) with $1.5 \mathrm{GHz}$ maximum bandwidth and $8 \mathrm{GSa} / \mathrm{s}$ maximum sample

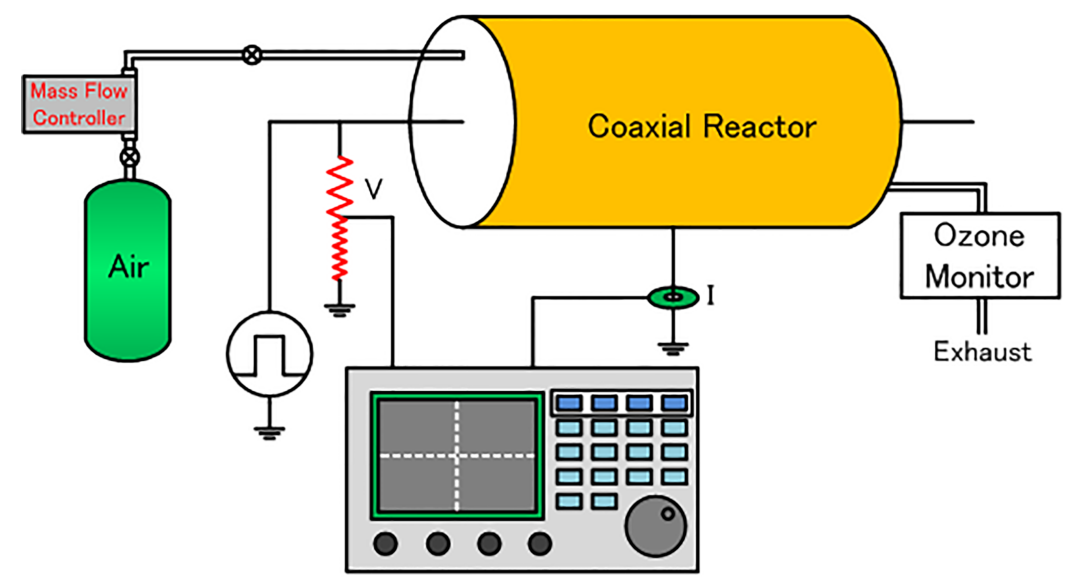

FIG. 2: Experimental setup for ozone generation 
rate. The input energy per pulse to the discharge electrode was calculated from the applied voltage and the discharge current: $\int$ (voltage $\times$ current $) d t$.

\section{RESULTS AND DISCUSSION}

\section{A. Output of Pulsed Power Generator}

Prior to the ozone generation experiment, a noninductive resistor was connected to the pulsed power generator as a matching load to confirm the output characteristics of the pulsed power generator. Figure 3 shows typical waveforms of (a) applied voltage to and (b) current through the $900 \Omega$ matching resistor. From Fig. 3(a) and (b), it is confirmed that the pulsed power generators produce rectangular pulses, and the pulse duration in half width at half maximum (HWHM) matches the theoretical values.

Figures 4 and 5 show waveforms of the applied voltages to and the discharge currents through the coaxial discharge reactor for the different pulse durations. Figures 4 and 5 were recorded at pulse repetition rates of 1 and 300 pps (pulses per second), respectively. At $1 \mathrm{pps}$, there is almost no ozone under the measurement device. At 300 pps, however, the concentration of ozone is $8.5 \mathrm{~g} / \mathrm{m}^{3}$. As such, the differences between waveforms are useful for studying ozone generation in this research.

The discharge phenomena of a positive pulsed discharge in coaxial electrode geometry has been investigated by Namihira et al. ${ }^{18}$ and Smulders et al. ${ }^{19}$ There are two discharge phases during application of pulsed voltage on the central wire electrode. Immediately after the pulsed voltage application, streamer heads are produced in the vicinity of the wire electrode and then propagate toward the ground cylinder electrode. This is called the streamer discharge phase and refers to the propagation period of the streamer heads between the electrodes. During the streamer discharge phase, the discharge current is small because of the large impedance of the capacitance between the

(a)

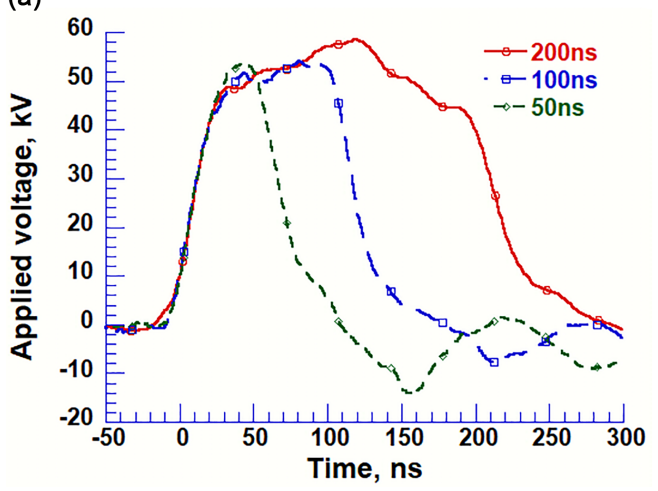

(b)

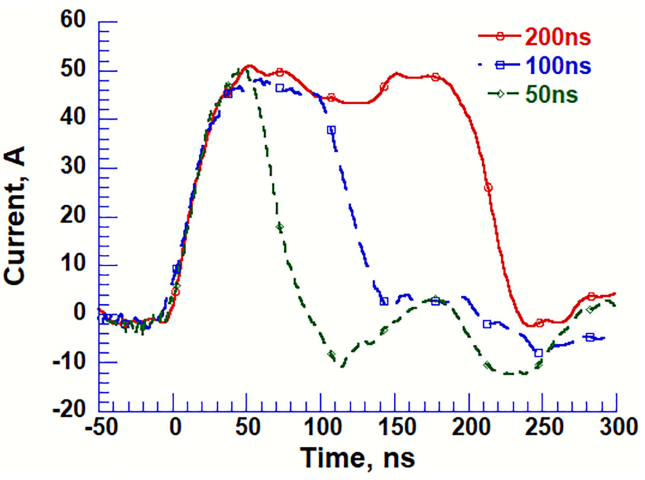

FIG. 3: Waveforms from pulsed power generator to a $900 \Omega$ matching resistive load. (a) Applied voltage. (b) Output current. 
(a)

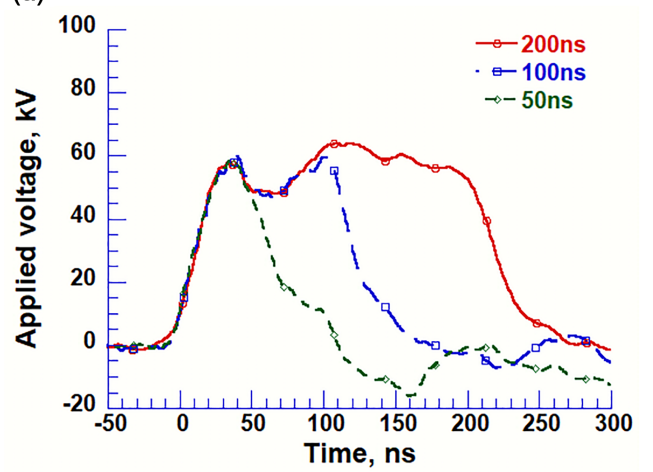

(b)

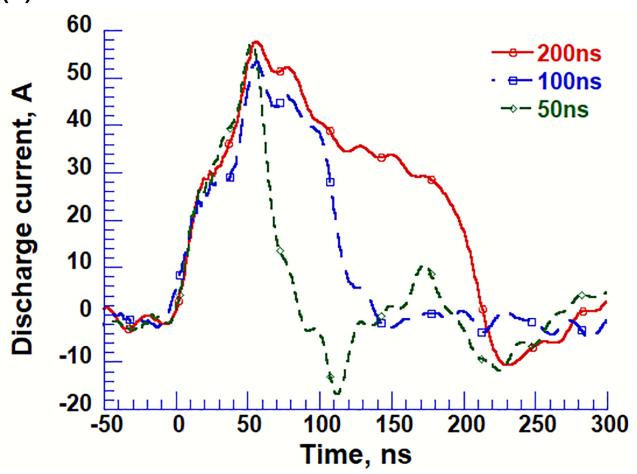

FIG. 4: Waveforms of applied voltage to and discharge current through ozone generation reactor at 1 pps. (a) Applied voltage. (b) Discharge current.

(a)

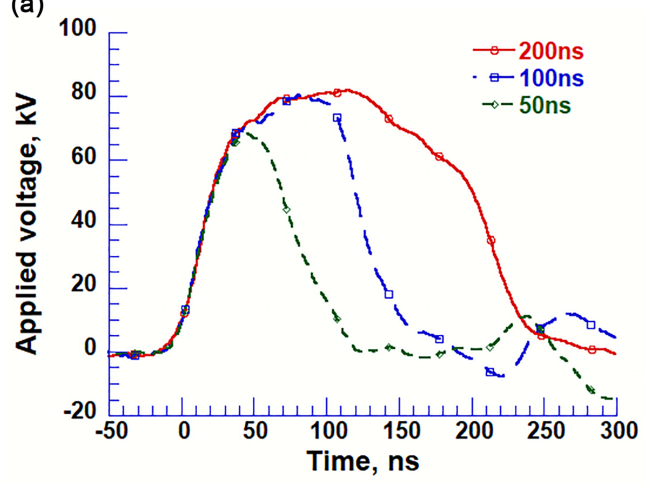

(b)

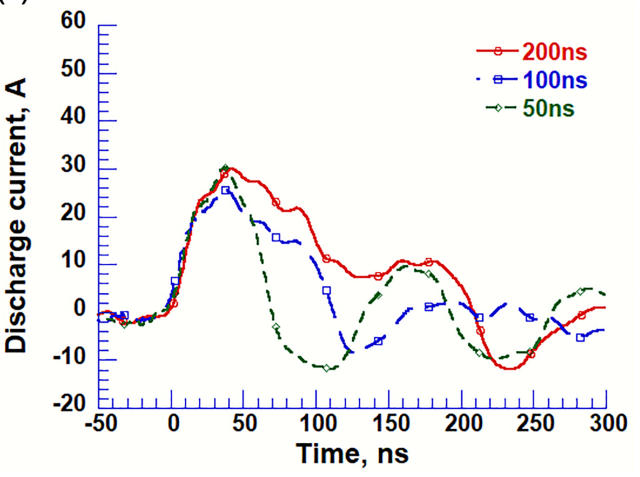

FIG. 5: Waveforms of applied voltage to and discharge current through ozone generation reactor at 300 pps. (a) Applied voltage. (b) Discharge current.

streamer heads and the ground electrode. Once the streamer heads reach the ground electrode, the discharge phase changes from streamer discharge to glow discharge. In the glow discharge phase, a large discharge current flows in the plasma column made by the streamer heads. As such, a pulsed discharge consists of two discharge phases: a streamer discharge and glow discharge. It is also known that the energies of energetic electrons during the streamer and glow discharges are around 5 to $10 \mathrm{eV}$ and 1 to $2 \mathrm{eV}$, respectively. ${ }^{20}$

As shown in Fig. 4(a) and (b), the applied voltage reached its peak value and the discharge current increased suddenly at $40 \mathrm{~ns}$, followed by a decrease in applied voltage at around $60 \mathrm{~ns}$. This is where discharge current reached its peak. This means that the impedance of discharge begins falling at $40 \mathrm{~ns}$, and streamer heads produced near the central wire electrode reach the ground cylinder electrode at $40 \mathrm{~ns}$. Therefore, $40 \mathrm{~ns}$ is 
understood to be the boundary between the streamer and the glow discharge phase. On the other hand, Fig. 5(a) and (b) show that the applied voltage increased and the discharge current decreased at $40 \mathrm{~ns}$, which is the opposite of the results shown in Fig. 4.

According to other studies ${ }^{21-23}$ ozone has the largest cross section of dissociative electron attachment at an electron energy range of 0 to $2.5 \mathrm{eV}$. This means that ozone has a strong influence on energetic electrons during the glow discharge phase (1 to 2 $\mathrm{eV})$. The data suggests that the current at the 300 pps pulse repetition rate decreased because electron attachment to ozone had already occurred after transitioning to the glow discharge phase.

Figure 6 shows the waveforms of the electrode impedances at different pulse durations. The impedance of the discharge electrode was calculated by dividing the applied voltage to the reactor by the discharge current through the reactor. In all of the charts in Fig. 6, the electrode impedances for each pulse repetition rate are almost the same until $40 \mathrm{~ns}$ (during the streamer discharge phase). After $40 \mathrm{~ns}$ (during the glow discharge phase), the electrode impedance at 1 pps decreased, and the impedances at higher pulse repetition rates increased. It was mentioned before that ozone has a strong attachment affinity for energetic electrons with electron energy of 0 to $2.5 \mathrm{eV}$. The energies of energetic electrons in the streamer discharge phase are 5 to $10 \mathrm{eV}$ and 1 to $2 \mathrm{eV}$ in the glow discharge phase. As such, electrode impedances during the streamer discharge phase exhibit no changes for the different pulse repetition rates because the energetic electron energy is high enough to prevent attachment to ozone molecules. On the other hand, during the glow discharge phase, the electrode impedances for higher pulse repetition rates increase because energetic electrons can attach to ozone molecules. At 1 pps, electrode impedance decreased because there is almost no ozone.

\section{B. Ozone Generation}

Figure 7 shows the input energy per pulse to the discharge reactor for pulse durations of 50 to 200 ns. Figure 7 shows that the input energy per pulse increased with the pulse duration at the same pulse repetition rate. At the same pulse duration, the input energy per

(a)

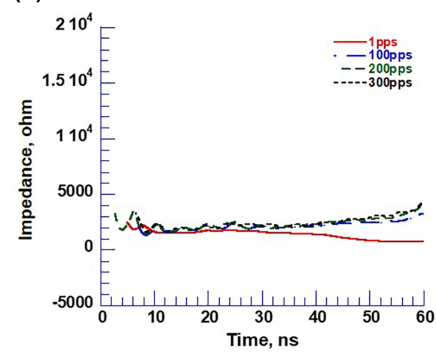

(b)

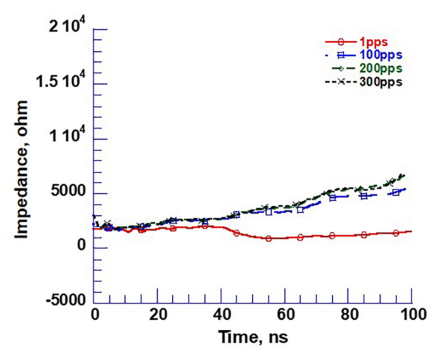

(c)

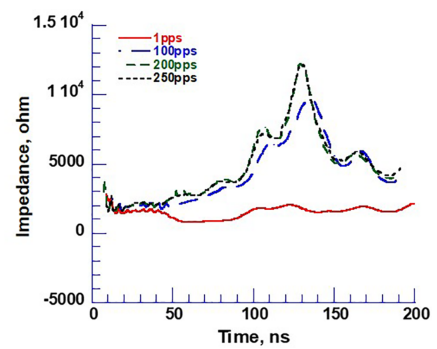

FIG. 6: Electrode impedances for different pulse repetition rates and different pulse durations. (a) 50 ns. (b) 100 ns. (c) 200 ns. 


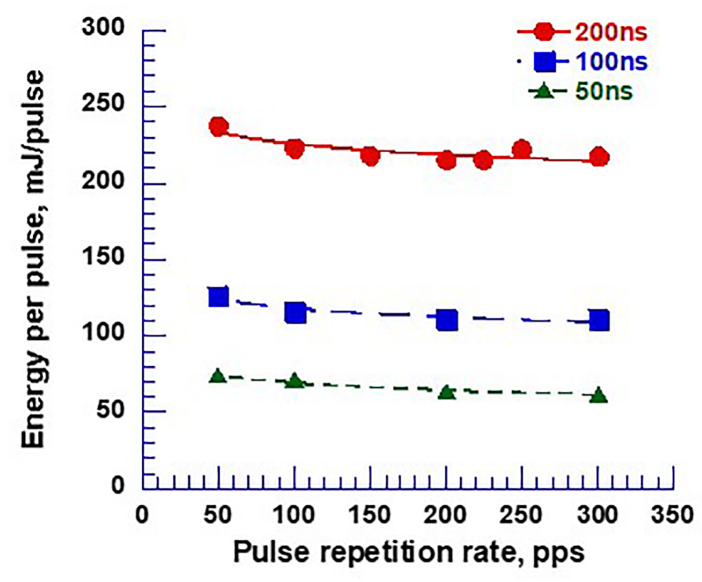

FIG. 7: Dependence of input energy per pulse on pulse repetition rate for different pulse durations

pulse is almost constant with increasing pulse repetition rate. As shown in Fig. 8, ozone concentration increases as pulse repetition increases, and the results in Fig. 7 indicate that ozone concentration is not affected by discharge states in the scope of this study.

Figure 8 shows the dependence of ozone concentration on the pulse repetition rate at different pulse durations. Generally speaking, ozone concentration increased as pulse repetition rate increased (input energy per unit time). In Fig. 8, for 200 ns pulse duration, ozone concentration increases with pulse repetition rate up to $150 \mathrm{pps}$ and then begins to decrease. For 100 ns pulse duration, ozone concentration increased with the pulse repetition rate until saturation at around $200 \mathrm{pps}$. By contrast, ozone concentration for $50 \mathrm{~ns}$ pulse duration increased with the pulse repetition rate and reached $8.5 \mathrm{~g} / \mathrm{m}^{3}$ at

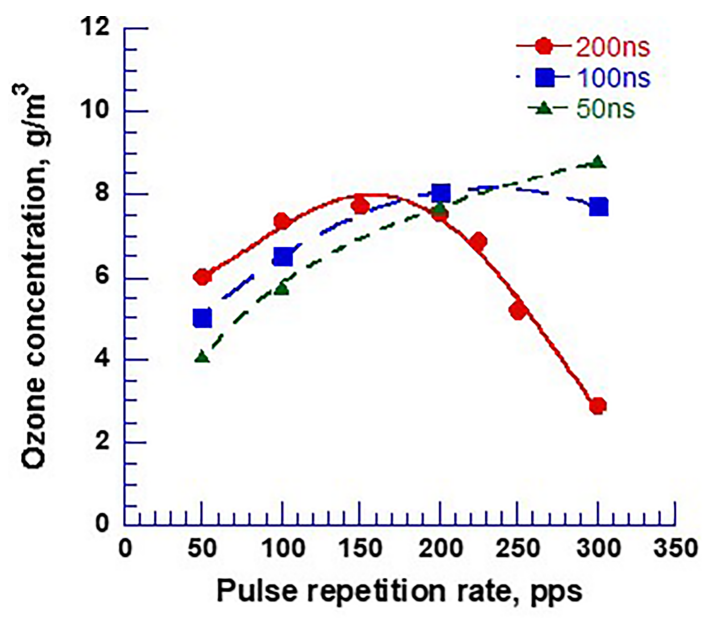

FIG. 8: Dependence of ozone concentration on pulse repetition rate for different pulse durations 
300 pps. Ozone production can be calculated by multiplying ozone concentration with seeding gas flow rate. Typically, ozone production for $50 \mathrm{~ns}$ pulse duration reached 0.51 $\mathrm{g} / \mathrm{h}$ at $300 \mathrm{pps}$.

Let us take a closer look at the differences in ozone generation characteristics for the different pulse durations. A positive pulsed discharge has two discharge phases: the streamer discharge phase and the glow discharge phase. In this study, the period of the streamer discharge phase is constant at around $40 \mathrm{~ns}$ for each pulse duration. However, the length of the glow discharge phase increases with the pulse duration. As stated before, because ozone molecules attach with lower energy electrons and dissociate during the glow discharge phase, ozone concentration decreased at a higher pulse repetition rate with a long pulse duration. It is also reported in the literature ${ }^{24}$ that the gas temperature during 100 ns of glow discharge increases to $400 \mathrm{~K}$, which is high enough to decompose ozone molecules. This means that the heat of the gas itself is also a reason for ozone degradation. We conclude from this information that saturation and the decrease of ozone concentration at longer pulse durations is caused by electron collision and thermal decomposition of ozone molecules, since the time domain of the glow discharge phase increases with longer pulse duration.

Figure 9 shows the dependence of ozone generation yield on pulse repetition rate for different pulse durations. Figure 9 illustrates that ozone yield decreases as pulse repetition rate and pulse duration increase. This occurs because the dissociation ratio of ozone molecules increases at higher pulse repetition rates and longer pulse durations. As a result, the shortest pulse duration offers the highest ozone generation yield.

Figure 10 is a characterization map of ozone generation using the pulsed discharges investigated in this study. The vertical axis is ozone generation yield, and the horizontal axis is ozone concentration. In Fig. 10, a well-performing ozonizer will offer results closer to the upper right sector of the graph. Figure 10 shows that shorter pulsed

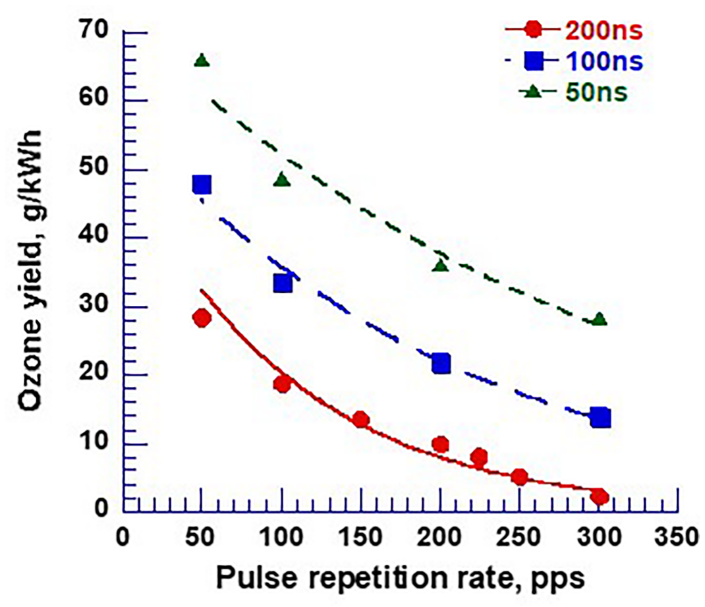

FIG. 9: Dependence of ozone yield on pulse repetition rate for different pulse durations 


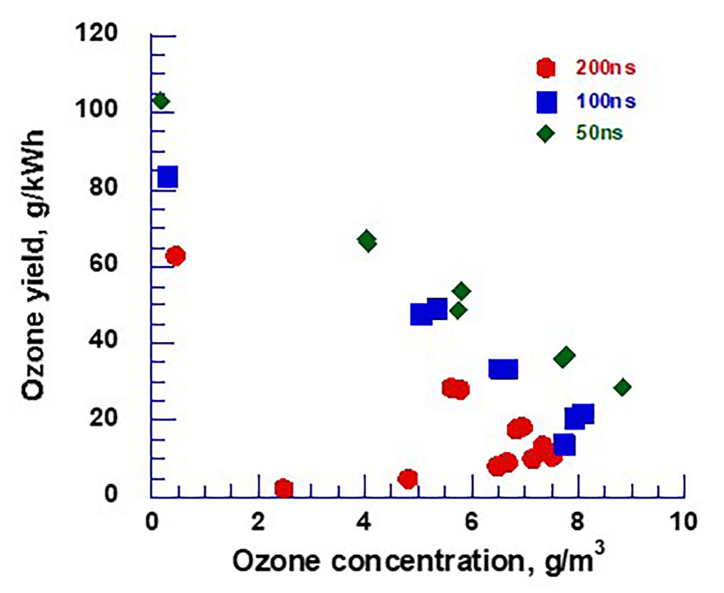

FIG. 10: Ozone characterization map for each pulsed discharge method

discharges lead to efficient ozone generation in an air-fed ozonizer. Of note is the fact that 200 ns results are nonlinear because ozone concentrations decrease at higher pulse repetition rates, as shown in Fig. 8.

\section{CONCLUSION}

In this study, pulsed discharges with three different pulse durations (50, 100, and $200 \mathrm{~ns})$ were used to generate ozone in an air-fed discharge reactor. Conclusions are as follows:

1. Investigating the output voltage and current waveforms revealed that ozone molecules attach with lower energy electrons and dissociate during the glow discharge phase, which has a lower electron energy than the streamer discharge phase.

2. With pulsed power, the glow discharge phase has a negative effect on ozone generation.

3. Shorter duration pulsed discharges yield higher energy efficiency in generating ozone by preventing the glow discharge phase.

\section{REFERENCES}

1. Elvis AM, Ekta JS. Ozone therapy: A clinical review. J Nat Sci Biol Med. 2011;2(1):66-70.

2. Pekárek S. Non-thermal plasma ozone generation. Acta Polytech. 2003;43(6):47-51.

3. Yuan D, Wang Z, Ding C, He Y, Whiddon R, Cen K. Ozone production in parallel multichannel dielectric barrier discharge from oxygen and air: The influence of gas pressure. J Phys D: Appl Phys. 2016;49(45):455203.

4. Yao S, Wu Z, Han J, Tang X, Jiang B, Lu H, Yamamoto S, Kodama S. Study of ozone generation in an atmospheric dielectric barrier discharge reactor. J Electrostat. 2015;75:35-42.

5. Jodzis S, Zięba M. Energy efficiency of an ozone generation process in oxygen. Analysis of a pulsed DBD system. Vacuum. 2018:155:29-37.

6. Zhang YF, Wei LS, Liang X, Deng HZ, Šimek M. Characteristics of the discharge and ozone generation

Volume 10, Issue 3, 2020 
in oxygen-fed coaxial DBD using an amplitude-modulated AC power supply. Plasma Chem Plasma Process. 2018;38:1199-208.

7. Chen HL, Lee HM, Chen SH, Chang MB. Review of packed-bed plasma reactor for ozone generation and air pollution control. Ind Eng Chem Res. 2008;47(7):2122-30.

8. Yehia A, Abdel-Salam M, Mizuno A. On assessment of ozone generation in DC coronas. J Phys D: Appl Phys. 2000;33(7):831.

9. Akiyama H, Sakugawa T, Namihira T, Takaki K, Minamitani Y, Shimomura N. Industrial applications of pulsed power technology. IEEE Trans Dielectr Electr Insul. 2007;14(5):1051-64.

10. Huiskamp T. Nanosecond pulsed streamer discharges Part I: Generation, source-plasma interaction and energy-efficiency optimization. Plasma Sources Sci Technol. 2020;29(2):023002.

11. Wang D, Namihira T. Nanosecond pulsed streamer discharges: II. Physics, discharge characterization and plasma processing. Plasma Sources Sci Technol. 2020;29(2):023001.

12. Okada S, Wang D, Namihira T, Katsuki S, Akiyama H. Effect of pulse width on oxygen-fed ozonizer. IEEJ Trans Fundam Mater. 2010;30(9):825-30.

13. Ma H, Qiu Y. A study of ozone synthesis in coaxial cylinder pulse streamer corona discharge reactors. Ozone Sci Eng. 2003;25(2):127-35.

14. Yagi S, Tanaka M. Mechanism of ozone generation in air-fed ozonisers. J Phys D: Appl Phys. 1979;12(9):1509-20.

15. Eliasson B, Kogelschatz U, Baessler P. Dissociation of $\mathrm{O}_{2}$ in $\mathrm{N}_{2} / \mathrm{O}_{2}$ mixtures. J Phys B: At Mol Phys. 1984;17(22):L797.

16. Samaranayake WJM, Miyahara Y, Namihira T, Katsuki S, Hackam R, Akiyama H. Ozone generation in dry air using pulsed discharges with and without a solid dielectric layer. IEEE Trans Dielectr Electr Insul. 2001;8(4):687-97.

17. Namihira T, Tsukamoto S, Wang D, Katsuki S, Hackam R, Akiyama H, Uchida Y, Koike M. Improvement of NOx removal efficiency using short-width pulsed power. IEEE Trans Plasma Sci. 2000;28(2):434-42.

18. Namihira T, Wang D, Katsuki S, Hackam R, Akiyama H. Propagation velocity of pulsed streamer discharges in atmospheric air. IEEE Trans Plasma Sci. 2003;31(5):1091-4.

19. Smulders EHWM, van Heesch BEJM, van Paasen SSVB. Pulsed power corona discharges for air pollution control. IEEE Trans Plasma Sci. 1998;26(5):1476-84.

20. Kanazawa S. Dynamics and structure of ignition process in plasmas. J Plasma Fusion Res. 2008;84(6):348-55.

21. Tegeder P, Kendall PA, Penno M, Mason NJ, Illenberger E. Electron stimulated desorption of $\mathrm{O}^{-}$and $\mathrm{O}_{2}^{-}$from condensed ozone: Possible implications for the heterogeneous photochemistry of stratospheric $\mathrm{O}_{3}$. Phys Chem Chem Phys. 2001;13:2625-9.

22. Walker IC, Gingell JM, Mason NJ, Marston G. Dissociative electron attachment (DEA) in ozone 0-10 eV. J Phys B: At Mol Opt Phys. 1996;29:4749-59.

23. Kuzumoto M. Extremely narrow discharge gap ozone generator. J Plasma Fusion Res. 1998;74(10):1144-50.

24. Okada S, Wang D, Namihira T, Katsuki S, Akiyama H. Spatiotemporal distribution of nitrogen rotational temperature during pulsed discharge in air. Jap J Appl Phys. 2011;50(8S1):08JB06. 